\title{
ALGUNOS ASPECTOS DE LA VEGETACION DE LAS SIERRAS MARTES Y AVE
}

\author{
R. FIGUEROLA
}

RESUMEN: Se comentan algunos aspectos sobre la fitosociología de los bosques y matorrales de las sierras Martés y Ave (Valencia, España) y se describen dos nuevas subasociaciones y seis variantes.

SUMARA: Some aspects about the woodland and scrub phytosociology of the Martés and Ave ranges (Valencia, Spain) are comented, also are described two new subassociation and six variants.

\section{INTRODUCCION}

El territorio que abarcan las sierras Martés y Ave se ubica en la parte centro-occidental de la provincia de Valencia, en una zona de tránsito entre la planicie litoral valentina y la Meseta Castellana y comprende una superficie de $600 \mathrm{~km}$ cuadrados aproximadamente que se enmarcan entre las siguientes coordenadas geográficas: $0^{\circ} 39^{\prime}$ y $1^{\circ}$ $6^{\prime}$ de longitud $W$ y $39^{\circ} 13^{\prime}$ y $39^{\circ} 24^{\prime}$ de latitud $N$. La zona queda igualmente enmarcada, en buena parte, por los ríos Jucar y Magro al sur y norte respectivamente.

A efectos corológicos el territorio pertenece al sector Setabense de la provincia corológica Valenciano-Catalano-Provenzal-Balear (Costa \& al., inéd.) y el ombroclima general es siempre seco oscilando las precipitaciones entre los 350 y $600 \mathrm{~mm}$ anuales. No obstante la topografía puede matizar localmente esta generalidad climática. Asi, en umbrías abrigadas pueden detectarse ombroclimas próximos al subhúmedo inferior $y$ en valles interiores abiertos otros que pueden referirse al semiárido superior.

El sustrato está constituido por materiales que pertenecen al Triásico, Terciario, Cretácico y Cuaternario, siendo los más importantes los que pertenecen a los períodos Cretácico y Terciario. Los afloramientos del Trías ocupan una parte importante en la zona NE y SW, presentando gran continuidad. Los depósitos cuaternarios, salvo afloramientos concretos poco representativos, quedan localizados en las cuencas de los ríos y adquieren verdadera importancia en la zona $\mathrm{N}$ a la orilla de los ríos Magro y Mijares.

De los cinco pisos bioclimáticos que Rivas-Martínez (1979:13; 1981:256; 1982a:277; 1982b:8) reconoce para la región mediterránea 
ibérica, solamente dos de ellos tienen representación en el territorio que estudiamos: Termomediterráneo y Mesomediterráneo. Para la zona el límite altitudinal entre ambos pisos puede establecerse alrededor de los 450-500 msm, según la exposición y el termoclimático en torno a los 15,5-16 $\mathrm{C}^{\circ}$ de media anual si bien la escasez de datos climáticos en el área no nos permite precisar con exactitud este último.

La vegetación potencial (clímax) del piso termomediterráneo es el carrascal termo-basífilo valenciano con acebuches y palmitos (Rubio longifoliae-Quercetum rotundifoliae Costa, Peris \& Figuerola 1982). La etapa madura del ecosistema climatófilo mesomediterráneo es el carrascal castellano-aragonés-valenciano sobre sustratos básicos (Bupleuro rigidi-Quercetum rotundifoliae $\mathrm{Br} .-\mathrm{Bl}$. \& Bolós 1957 em. nom. Rivas-Martínez 1982).

\section{MATORRALES SERIALES}

Las comunidades camefíticas, eventualmente nanofanerofíticas (tomillares y romerales), son las formaciones vegetales más extendidas y las que mejor representan el paisaje vegetal de ambas sierras. Su carácter de etapa de sustitución de los bosques climácicos evidencia la degradación de estos. La variabilidad ecológica y florística de los matorrales de la zona puede concretarse en las siguientes asociaciones:

\section{Helianthemo-Thymetum piperellae Rivas-Goday 1958}

Sinestructura y sinecología: Matorral de aspecto muy variable que, según las condiciones, puede presentarse en facies de tomillar, romeral o ahulagar. Coloniza suelos básicos (calizas cretácicas y miocenas) no margosos y su óptimo bioclimático se encuentra en los horizontes inferior y medio del piso mesomediterráneo.

Dinamismo: En la serie dinámica ocupa una posición intermedia entre los coscojares del Rhamno-Cocciferetum Br.-Bl. \& Bolós 1957 (etapa preforestal de los carrascales climácicos) y los pastizales vivaces del Teucrio-Brachypodietum ramosi Bolós 1957 o Phlomido-Brachypodietum ramosi $\mathrm{Br} .-\mathrm{Bl} .1924$.

Variabilidad: en función de la variabilidad ecológica y florística hemos observado las sigientes variantes de la subasociación típica:

var. de Erica terminalis (Tab. 1, inv. 2). Corresponde a ecótopos pedregosos con escorrentías superficiales.

var. de Ononis fruticosa (Tab. 1, inv. 3). Matiza sustratos margosos bajo condiciones de ombroclima seco.

var. de Cistus salvifolius (Tab. 1, inv. 4). Denuncia suelos descarbonatados en superficie. Esta variante corresponde a la descrita por Costa \& al. (1982: 45).

var. de Ononis aragonesis (Tab. 1, inv. 5). Pone de manifiesto la influencia maestracense que se aprecia en algunas umbrías.

var. de Genista mugronensis (Tab. 1, inv. 6). Revela la influencia manchega que se aprecia en la parte occidental del territorio y er ałgunas exposiciones a solana. 
var. de Chronanthus biflorus (Tab. 1, inv. 7). Matiza sustratos margosos bajo condiciones ombroclimáticas cercanas al semiárido.

Anthyllido-Cistetum clusii Br.-Bl. 1935

Sinestructura y sinecología: Niatorral de talla mediana en el que puede dominar Cistus clusii, Anthyllis cytisoides o Rosmarinus officinalis. Coloniza sustratos margosos del Trías y rara vez supera el horizonte inferior mesomediterráneo.

Dinamismo: En las series dinámicas antecede al Rhamno-Cocciferetum mesomediterráneo o al Querco-Lentiscetum Br.Bl. \& al. 1935 em. A. \& O. Bolós 1950 termomediterráneo. Matiza las subseries edáficas sobre sustratos margosos.

Variabilidad: El contacto de esta asociación con el matorral gipsicola (Gypsophilo struthii-Ononidetum edentulae Costa, Peris \& Figuerola inéd.) se verifica por medio de la subasociación ononidetosum edentulae Costa, Peris \& Figuerola subas. nova (Tab. 2, invs. 3 y 4 , syntypus $n^{\circ} 3$ ) en la que Ononis tridentata var. edentula y Gypsophila struthium son diferenciales.

\section{VEGETACION POTENCIAL TERMOMEDITERRANEA}

La etapa madura del ecosistema vegetal termomediterráneo es un carrascal denso (Rubio longifoliae-Quercetum rotundifoliae) caracterizado por nanofanerófitos térmicos que actuan como diferenciales frente al carrascal mesomediterráneo. Los más representativos y fieles son: Chamaerops humilis y Osyris quadripartita. Rubia peregrina subsp. longifolia, aunque tiene su óptimo en este bosque, llega hasta el horizonte inferior mesomediterráneo donde matiza los carrascales de transición.

Cuando el ombroclima se aproxima al subhúmedo el carrascal se enriquece en elementos mesofíticos (Fraxinus ornus, Viburnum tinus, etc.) que matizan la subasociación fraxinetosum orni Costa, Peris \& Figuerola 1982. Esta es la etapa madura de la subserie termo-subhúmeda de la carrasca.

La degradación del carrascal termomediterráneo origina por dinamismo una comunidad vegetal que, probablemente, es la que mejor representa el paisaje vegetal de las áreas térmicas valencianas: el matorral de coscojas y lentiscos (Querco-Lentiscetum Br.-Bl. \& al. 1935 em. A. \& O. Bolós 1950). Este matorral es la etapa madura de las áreas termomediterráneas semiáridas y representa la vegetación permanente de crestas y ecótopos con poco suelo en el dominio del carrascal.

\section{VEGETACION POTENCIAL MESOMEDITERRANEA}

La etapa madura del ecosistema vegetal mesomediterráneo es un carrascal (Bupleuro rigidi-Quercetum rotundifoliae) sensiblemente más pobre en especies que el anterior. Las lianas (Smilax aspera, Lonicera implexa, Clematis flammula), abundantes en el carrascal termomediterráneo, son bastante escasas en el mesomediterráneo. Otra caracte- 
rística relevante del bosque potencial mesomediterráneo es su pauciestratificación frente a la pluriestratificación que ostenta el termomediterráneo. En la tabla 3 se presentan seis inventarios que dan idea sobre la composición florística y variabilidad del carrascal mesomediterráneo.

En las áreas de transición al piso termomediterráneo (horizonte inferior mesomediterráneo) la presencia de ciertos elementos térmicos permite reconocer la subasociación pistacietosum lentisci Rivas-Martínez subas. nova (invs. $3-6$, syntypus $n^{\circ} 5$ ). Son características diferenciales Pistacia lentiscus y Rubia peregrina subsp. longifolia. Esta subasociación es la etapa madura de la subserie termófilo-levantina del carrascal mesomediterráneo y matiza, en bastantes ocasiones, el tránsito entre las provincias corológicas Valenciano-Catalano-Provenzal-Balear y Castellano-Maestrazgo-Manchega.

La alteración del bosque potencial mesomediterráneo permite la instalación de coscojares (Rhamno-Cocciferetum) que actúan como etapa preforestal del carrascal en territorios con ombroclima seco. Si el ombroclima tiende al semiárido estas garrigas representan la etapa madura del ecosistema vegetal mesomediterráneo.

\section{BIBLIOGRAFIA}

COSTA, M. \& al. -inéd.- Ensayo corológico de la región valenciana.

COSTA, M., PERIS, J.B. \& FIGUEROLA, R. -1982- Sobre los carrascales termomediterráneos valencianos. Lazaroa, 4: 37-52.

RIVAS-MARTINEZ, S. -1979- Brezales y jarales de Europa Occidental (Revisión fitosociológica de las clases Calluno-Ulicetea y Cisto-Lavanduletea). Lazaroa, 1: 5-127.

RIVAS-MARTINEZ, S. -1981- Les étages bioclimatiques de la végétation de la Péninsule Ibérique. Anal. Jard. Bot. Madrid, 37 (2): 251-268.

RIVAS-MARTINEZ, S. -1982a- Etages bioclimatiques, secteurs chorologiques et séries de végetation de l'Espagne méditerranéenne. Ecol. Med., $8(1-2): 275-288$.

RIVAS-MARTINEZ, S. -1982b- Memoria del Mapa de las Series de Vegetación de la provincia de Madrid. Diputación de Madrid. 
Tabla 1

HELIANTHEMO-THYMETUM PIPERELLAE Rivas-Goday 1958

(Rosmarino-Ericion, Rosmarinetalia, Ononido-Rosmarinetea)

$\begin{array}{llllllll}\text { Número de inventario } & 1 & 2 & 3 & 4 & 5 & 6 & 7 \\ \text { Altitud }(1=10 \mathrm{~m}) & 72 & 80 & 85 & 70 & 92 & 96 & 50 \\ \text { Exposición } & \mathrm{E} & \mathrm{NE} & \mathrm{S} & \mathrm{NW} & \mathrm{NE} & \mathrm{S} & \mathrm{NE} \\ \text { Cobertura }(\%) & 60 & 80 & 60 & 60 & 80 & 50 & 80\end{array}$

Características territoriales de asociación y alianza:

$\begin{array}{llllllll}\text { Thymus piperella } & 2.2 & 2.2 & 2.2 & 2.2 & 2.2 & 2.2 & 1.2 \\ \text { Helianthemum cinereum } & 1.1 & 1.1 & +.1 & 1.1 & . & +.2 & 1.1 \\ \text { Ulex parviflorus } & 2.2 & 2.2 & 3.3 & 3.3 & +.2 & 1.1 & . \\ \text { Erica multiflora } & 2.2 & 2.2 & 1.2 & 1.2 & 3.2 & 2.3 & . \\ \text { Fumana ericoides } & 1.1 & 1.1 & 1.1 & 1.1 & +.1 & 1.1 & \text {. }\end{array}$

Diferenciales:

Erica terminalis

Ononis fruticosa

Cistus salvifolius

Ononis aragonensis

Genista pumila

subsp. mugronensis

Chronanthus biflorus

Características de orden y clase:

\begin{tabular}{|c|c|c|c|c|c|c|c|}
\hline Rosmarinus officinalis & 3.3 & 1.2 & 3.2 & 3.3 & 2.2 & 1.2 & 2.2 \\
\hline Helianthemum lavandulifolium & 1.2 & 1.1 & 1.1 & . & +.1 & 1.2 & . \\
\hline Thymus vulgaris & 1.2 & . & . & 1.2 & . & +.2 & 1.2 \\
\hline Atractylis humilis & 1.1 & . & 1.1 & . & . & . & 1.1 \\
\hline Genista scorpius & +.2 & . & . & +.2 & . & . & 1.2 \\
\hline \multicolumn{8}{|l|}{ Compañeras: } \\
\hline Brachypodium retusum & 2.2 & 2.2 & 1.2 & 2.3 & 3.3 & 2.3 & 3.2 \\
\hline Helictotrichum filifolium & . & 1.2 & 1.2 & . & +.2 & 1.2 & . \\
\hline Koeleria vallesiana & 1.2 & . & . & 1.2 & $\therefore$ & 1.1 & +.1 \\
\hline Dorycnium pentaphyllum & +.2 & . & . & +.2 & 1.2 & . & . \\
\hline Carex halleriana & 1.2 & 1.2 & 1.1 & . & . & . & . \\
\hline
\end{tabular}

Localidades y especies accidentales: 1. Entre la Portera y los Pedrones. 30SXJ65. Cuscuta epithymum, Linum suffruticosum subsp. suffruticosum, Eryngium campestre, Bupleurum fruticescens. 2. Pista Forestal de Subida al Martés. 30SXJ75. Centaurium barrelieri, Cephalaria leucantha, Sideritis incana subsp. incana, Lithodora fruticosa, Satureja cuneifolia subsp. gracilis. 3. Venta Gaeta. 30SXJ75. Euphorbia falcata subsp. rubra, Teucrium polium subsp. expansum, Fumana laevis, Asperula cynanchica, Bupleurum rigidum. 4. Muela de Dos Aguas. 30SXJ85. Scabiosa stellata, Leuzea conifera, Fumana glutinosa, Asperula cynanchica, Eryngium campestre, Cistus albidus. 5. Alta umbría del Martés. 30SXJ75. Solidago virgaurea, Globularia valentina, Teucrium polium subsp. aureum, Daphne gnidium, Rubia peregrina, 6. Solana de la Sierra Martés. 30SXJ75. Convolvulus lanuginosus, Arenaria aggregata subsp. aggregata, Helianthemum croceum, Paronychia aretioides, Festuca hystrix, Arenaria obtusiflora subsp. obtusiflora, Phlomis crinita, Euphorbia minuta, Bupleurum fruticescens, Sideritis incana subsp. incana. 7. Valle de Cofrentes. 30SXJ64. Cistus clusii, Fumana glutinosa, Teucrium capitatum, Linum narbonense, Dactylis glomerata subsp. hispanica. 
Tabla 2

ANTHYLLIDO-CISTETUM CLUSII Br.-Bl . 1935

(Rosmarino-Ericion, Rosmarinetalia, Ononido-Rosmarinetea)

Número de inventario

Altitud $(1=10 \mathrm{~m})$

$\begin{array}{llll}1 & 2 & 3 & 4 \\ 45 & 39 & 38 & 39 \\ \mathrm{~S} & \mathrm{SE} & \mathrm{SE} & \mathrm{N} \\ 75 & 75 & 50 & 50\end{array}$

Exposición

$\begin{array}{llll}3.3 & 3.3 & 2.2 & 2.1\end{array}$

Anthyllis cytisoides

$\begin{array}{llll}2.2 & +.2+.2 & +2.1\end{array}$

Cistus clusii

$\begin{array}{llll}2.2 & +.2 & +.2 & 2.1 \\ 1.1 & +.2 & . & +.1\end{array}$

Diferenciales:

Ononis tridentata var. edentula

Gypsophila struthium

$$
\begin{array}{lll}
. & 2.2 \quad 2.3
\end{array}
$$

Características de orden y clase:

Rosmarinus officinalis

Thymus vulgaris

Fumana glutinosa

Atractylis humilis

Compañeras:

Brachypodium retusum

Eryngium campestre $\begin{array}{llll}2.2 & 3.3 & 1.1 & +.2\end{array}$

$\begin{array}{llll}1.1 & 1.1 & 1.1 & 1.1\end{array}$

$\begin{array}{lll}1.1 & 1.1 & 1.1\end{array}$

$+.1 \quad 1.1 \quad . \quad+.1$

Localidades y especies accidentales: 1. Entre Yátova y La Portera. 30SXJ 86. Erica multiflora, Phagnalon rupestre, Convolvulus lanuginusus, Helianthemum pilosum, Genista scorpius, Helianthemum cinereum, Stipa tenacissima, Dorycnium pentaphyllum. 2. Valle de Cofrentes. 30SXJ64. Erica multiflora, Phagnalon rupestre, Genista scorpius, Teucrium capitatum, Argyrolobium zannoni, Lavandula latifolia, Stipa offneri, Hyparrhenia hirta, Sedum sediforme, Phlomis lychnitis, Dactylis glomerata subsp. hispanica, Phlomis crinita, Aristolochia pistolochia, Avenula bromoides, Psoralea bituminosa. 3. Salto de Cofrentes. 30SXJ64. Convolvulus lanuginosus, Stipa tenacissima, Stipa offneri, Hyparrhenia hirta, Sedum sediforme, Plantago albicans, Sanguisorba minor. 4. Salto de Cofrentes. 30SXJ64. Helianthemum pilosum, Teucrium capitatum, Dorycnium pentaphyllum, Phlomis lychnitis, Centaurea aspera subsp. stenophylla. 
BUPLEURO-QUERCETUM ROTUNDIFOLIAE Br.-Bl. \& O. Bolós 1957 em. nom. Rivas-Martínez 1982

(Quercion ilicis, Quercetalia ilicis, Quercetea ilicis)

\begin{tabular}{|c|c|c|c|c|c|}
\hline Número de inventario & 1 & 2 & 3 & 4 & 5 \\
\hline Altitud $(1=10 \mathrm{~m})$ & 72 & 63 & 46 & 46 & 47 \\
\hline Exposición & W & E & $\mathrm{E}$ & $\mathrm{NE}$ & $\mathrm{E}$ \\
\hline Cobertura (\%) & 90 & 90 & 80 & 60 & 80 \\
\hline
\end{tabular}

Caracteristicas territoriales de asociación y unidades superiores:

\begin{tabular}{|c|c|c|c|c|c|c|}
\hline Quercus rotundifolia & 4.4 & 5.5 & 3.3 & 3.3 & 3.3 & 4.3 \\
\hline Quercus coccifera & 1.2 & +.2 & 1.2 & 1.2 & +.2 & 1.2 \\
\hline Daphne gnidium & . & . & 1.2 & 1.2 & 1.2 & 1.1 \\
\hline Rubia peregrina subsp. peregrina & 1.1 & 2.1 & . & . & . & 2.1 \\
\hline Juniperus oxycedrus & 1.2 & $1 \cdot 2$ & - & . & . & +.2 \\
\hline \multicolumn{7}{|l|}{ Diferenciales } \\
\hline Rubia peregrina subsp. longifolia & . & . & $1 \cdot 2$ & 1.1 & 1.1 & +.1 \\
\hline Pistacia lentiscus & - & - & $2 \cdot 2$ & 2.2 & 2.2 & 2.2 \\
\hline \multicolumn{7}{|l|}{ Compañeras: } \\
\hline Brachypodium retusum & 3.3 & 3.3 & 2.3 & 3.3 & 3.3 & 3.3 \\
\hline Ulex parviflorus & +.2 & +.2 & +.2 & +.2 & +.2 & 1.2 \\
\hline Teucrium chamaedrys subsp. pinnatifidum & +.1 & 1.1 & +.1 & - & 1.1 & 1.1 \\
\hline Erica multiflora & $\cdot$ & · & +.1 & +.2 & . & 1.1 \\
\hline Rosmarinus officinalis & +.2 & +.2 & $\cdot$ & · & +.1 & - \\
\hline Echinops ritro & +.1 & +.1 & - & . & . & +.1 \\
\hline
\end{tabular}

Localidades y especies accidentales: 1. La Cabezuela. 30SXJ65. Dorycnium pentaphyllum, Carex halleriana, Thymus vulgaris, Genista scorpius, Atractylis humilis. 2 Entre La Cabezuela y Castilblanques. 30SXJ65. Cistus salvifolius, Genista scorpius. 3. Muela de Dos Aguas. 30SXJ85. Rhamnus alaternus, Elaeoselinum asclepium subsp. meoides, Thymus piperella. 4. Muela de Dos Aguas. 30SXJ85. Asparagus stipularis, Asparagus acutifolius, Elaeoselinum asclepium subsp. meoides, Saponaria ocymoides. 5. Muela de Dos Aguas. 30SX J85. Asparagus stipularis. 6. Muela de Dos Aguas. 30SXJ85. Polygala rupestris. 